\title{
Applied Research of Cloud Computing on the Sharing of Educational Information Resource
}

\author{
Gang Zhao, Chen LiJuan, Ye QiuXu \\ College of Information Technology, Journalism and Communications, \\ Huazhong Normal University, \\ Wuhan, Hubei ,China. \\ zhaogang@mail.ccnu.edu.cn,1071776299@qq.com,290662267@qq.com
}

\begin{abstract}
The sharing and optimized configuration of educational information resources is very important of the realization of fair education. Cloud Computing is a new service computing model, which can integrate, manage and allocate the computing resources distributed on the Internet. In this paper, we discussed with the apply of cloud computing to the sharing of educational resources. The way and the benefit of the cloud computing applying are very carefully investigated. Supplementary strategies of applying cloud computing to solve remained problems are also proposed.
\end{abstract}

Keywords - Cloud Computing, Educational Information Resource, Sharing and Configuration.

\section{INTRODUCTION}

The differentiated development of the economic between different regions in China, the unbalanced characteristics of the distribution of educational information resources between the different regions, between the city area and rural area in the same region, between key schools and non-key schools become more and more obviously. The lack of educational information resources in underdeveloped areas and the wrong configuration of high quality educational resources have led to the unfair phenomenon of educational systems ${ }^{[1]}$. The Chinese government has recognized improving the fair of education system as the basic education policy of the whole country. It also clamed that the fundamental ways to lead to fair education is reasonable configuration of educational resources. And the information technology is a revolutionary factor for the education development. The way how to use the information technology to the sharing and optimized configuration of educational information resources become a hot research topic.

Cloud Computing is a new service computing model, which can integrate, manage and allocate the computing resources distributed on the Internet ${ }^{[2]}$. The cloud computing provides service for vast amount users with a united interface . Data-centered, service-centered and user-

This work is supported by the Project named Research on Educational Information Resource Sharing and Optimal Configuration based on Cloud Computing granted by the Humanities and Social Science project of Chinese Ministry of Education with Grant No. 11YJA880163. centered is the characteristics of cloud computing. All data, software and services are located on the cloud. So cloud computing provides a new method and way for the sharing services of educational information resources. It may give better configuration of education resources. In this paper we try to introduce cloud computing architecture and technology to the sharing of educational information resources. Our research at first finds out the problems and obstruction for educational resource sharing and then investigated the possibility of the cloud computing technology to solve them. We proposed some strategies and method to maximum the advantage of cloud computing based on pervious analysis.

The remainder of this paper is organized as follows. In Section 2 we analyze the current status and problems of the sharing of educational information Resource. Then Section 3 introduces the basic concept and characters of cloud computing technology. The advantages and disadvantages are deeply discussed in Section 4. The strategies and applying issues of cloud computing are carefully studied in Section 5.

\section{STATUS ANALYSIS OF EDUCATIONAL INFORMATION RESOURCES SHARING}

With the rapid development of IT industry, joint construction and sharing of information resources has attracted the attention. Library, for example, joint construction and sharing of instructional resources from one school multi-campus coordinated loaning and returning to build more schools shared system and then to now, regional library consortium. All these make the breadth and depth of joint construction and sharing expand gradually; especially the representative building projects Chinese safe literature system of higher education (CALIS) and the National Science and Technology Library Document Center (NSTL) are successful examples of realizing the sharing of information resources. These projects have greatly improved the resource status of the library, saved the cost, the construction and the development and utilization of resources and mechanism innovation, have immeasurable social benefits. However, the educational information resource co-construction and sharing still exists many 
problems which can be categorized into two aspects: technical problems and economics problems.

\section{A. Technical problems}

At present the construction of self-built educational database lack of unified planning and coordination, the majority of existing database are small ,low degree of standardization, and the quality is not satisfied. Educational resources sharing is often limited in some areas of the same interface data resources.

The educational resources repositories may be widely distributed and managed by different education organizations developed with different software technologies. They are either closed systems or systems that allow user access only through proprietary interfaces. The lack of interoperability between these system leads to isolated information islands. Users can hardly find out their interested learning resources in such a distributed and isolated environment.

Educational information resources must be corrective, adaptive, prevention and improvement of maintenance in the whole life cycle, they need to bring forth the new through the old and keep pace with times. With the application of multimedia and new technology, the types of Educational resources expanded rapidly. Data structure is becoming more and more complex, the amount of data expanded rapidly. All of these put forward higher requirements. However, the different resources construction teams have different abilities.

Even the Educational information resource repositories are developed with the same technology, different technical maintenance abilities in different education organizations also prevent the sharing.

In general , low degree of standardization, lack of interoperability between educational resource repository systems and different technical maintenance abilities keep educational information resources back from high-level sharing and exchanging.

\section{B. Economics problems}

Though "School-to-School" system is in development, but has no breakthrough in China. The thought of people are still unchanged and sharing consciousness is not enough. High quality educational resources of colleges and universities are usually hung on the inner campus, for external users have many limitations, resulting in unnecessary waste of resources.

The development of high quality educational resources may spend much money. If there is no mechanism to guarantee the original investment, no leader or manager of the education organizations dare to share the educational resources with other organizations or individuals.

In addition, technical maintenance cost is another economic factor to prevent the sharing of educational information resources. In the education organizations with high quality resources, there often exists a good technical support team. The higher the level of the resources is the more the cost of the labor power and accessory is. However, in the education organizations with low quality resources, the technical supporting team may just have one person who always is a teacher for information technology course .This phenomenon often happens in the rural area.

Finally, the information technology infrastructures for the educational resources also require vast investment. Most education organizations cannot bear the large investment for the hardware and software to provide the educational resource service. However, there is still a strange pheromone. Redundant construction of educational resources is fairly common problems in many education organizations although they only have very limited budget. Less education organizations want to build a public educational resource repository.

In general, the cost in the life cycle including development, maintenance and service providing of education resources is the obstacle of the sharing.

\section{CHARACTERISTICS OF CLOUD COMPUTING}

Cloud computing is based on the Internet. The cloud is composed of numerous computers, providing various kinds of service for the client. The client is connected with the cloud by the Internet. The biggest breakthrough of Cloud computing is not in the technical innovation but in the change of the concept about IT. Cloud computing platform put IT resources into package and provides uses resources with interface or browser.

The type of service of cloud computing can be divided into three different levels: infrastructure as a Service,platform as a Service,software as a Service ${ }^{[3]}$. The IaaS supports PaaS and SaaS, and PaaS can provide service for SaaS. At the same time, every cloud computing level can provide users with services independently.

\section{A.Infrastructure as a Service}

IaaS is located in the bottom of the three layers of cloud computing service, which provides the basic computing and storage capacity ${ }^{[4]}$. For an example, just like computing capacity, the basic unit of providing services is the server, including CPU, memory, storage, operating system and software. Automation and virtualization is the core technology of this layer service. The technology of automation enables users get the resources by themselves. Virtual technology can improve resource utilization, reduce IaaS platform cost and user using cost by sharing physical resources. The dynamic transfer function of virtualization can bring greatly improve service availability.

\section{B.Platform as a Service}

PaaS is located in the middle layer of cloud computing. Its function is equivalent to cloud' s operating system, and is the core of cloud computing ${ }^{[5]}$. It provides the end user with application development environment based on Internet, including the application programming interface 
and running platform. It can support users a variety of hardware and software resources, which can be used in the whole life' s creation. What the service provider is the IT ability or some logic resources, such as database, file system and applications running environment.

\section{C.Software as a Service}

SaaS is directly to end users and each enterprise. The applications are accessible from various client devices through either a thin client interface, such as a web browser (e.g., web-based email), or a program interface ${ }^{[5]}$. The service provider is responsible for the maintenance and management of software and hardware facilities, and users can get sources free (providers can benefit from such ads project) or on-demand leased way. SaaS support applications installation, updating and maintenance of software, which are completed on the cloud.

The core of cloud computing is computing-centered and data storage-centered . It is a computer group which is made of hundreds or thousands or even millions of computers, the aggregation and distribution of information, and provides service to the customer through the network. In this way, users only need to use the computers, mobile phones, PDA and other devices to access the Internet and get necessary information service .The requirement of client hardware is low in cloud computing. You can get the resources as long as you have an access to the Internet.

\section{BENEFIT OF CLOUDING COMPUTING APPLING TO EDUCATIONAL RESOURCES SHARING}

According to the problems mentioned in Section 2, considered the architecture and characteristics of cloud computing, we investigated whether the Cloud computing can solve part of the problems, while other parts cannot be solved and even new problems are raised.

We can find cloud computing is very suitable for tackle the technical problems such as low degree of standardization, lack of interoperability between educational resource repository systems and different technical maintenance abilities. At first in cloud computing model we can design a virtual educational information resources repository. An all data is uploaded and storage into this virtual repository. Although the real repositories consist of the virtual repository may distribute on different place and organization, the cloud computing technology ensures much of the difference between the real repositories is transparent to both the users and the system managers. So standardization and interoperability are improved. In additional, there is only a high-level technical maintenance team required in the cloud computing center and less or no technical main tenant team on the real repositories' side.

For the economic problems that prevent resource sharing, cloud computing can solve part of them. The cost of maintenance and service providing of education resources for all the involved education organizations can be indeed decreased because there is just only a cloud- computing center. Both the maintenance investment and the information technology infrastructure investment can just be used to the center. The utilization ratio of the investment can be increased.

When we investigated the cloud computing applied to the sharing of educational resources, some additional advantages we can got.

\section{A.infrastructure utilization ratio is improved:}

As mentioned in the start of section 4 , cloud computing can integrate many high quality educational information resources of domestic and international organizations, and form a large scale, professional discipline, curriculum of resources repositories, all the resources in cloud computing are put in the cloud, so that every user can have access to the resources that they need through the browser. Of course the same resources do not need to put in the cloud. The infrastructure of these resources can be used in other places, for example, to allow users to store their resources and so on, so as to improve the utilization of infrastructure.

\section{B.The security of information resources are improved}

Educational resources in Colleges and universities is storage on many servers in a cluster of cloud computing network, we don't have to worry about data security problems like negligence of lost data, hardware damage or computer virus and hacker intrusion ${ }^{[6]}$.We will store the data that we often used to our hard disk (U disk, mobile hard disk and so on), but it is easy to cause the loss of data. Cloud storage services take the data security risk that we will face into the "cloud" network server so that the information security is greatly improved.

\section{C.The management and system maintenance level has been improved}

Information storage and management together is convenient for professional team to take professional security measures and means, and it can avoid data loss and damage which is caused by personal reasons (such as viruses, Trojan invasion, the damage of equipment).In addition, redundancy, fault-tolerant mechanism of cloud storage services can ensure the data security.

However, the cloud computing technology cannot solve some problems and the introduce of this technology raised new problems. The investment of the development of high quality educational information resources is still very high. And the education organizations developing high quality educational resources cannot get any compensation from the sharing via the cloud computing.

\section{SUPPLEMENTARY STRATEGIES OF APPLYING CLOUD COMPUTING TO EDUCATIONAL INFORMATION RESOURCES}

As discussed in Section 4, cloud computing in summary can improve the sharing of educational resources but some problems remains unsettled. So new strategies of 
applying cloud computing should be designed. We proposed three strategies here.

The first strategy is to design a new service pattern for educational information resource based on Cloud Computing. The profit of all participator in the resource consumption chain including the government, educational information resource provider, educational information resource service provider and users should be guaranteed. The information flow, control flow and resource flow become very complex in such Cloud Computing Model, some standards and supervised specification should also be carefully designed.

The second strategy is to make a mechanism to both protect right and interest of educational information resources and inspire the sharing and exchanging. For the former one, resource rights control and public retrieval mechanism should be apply during the sharing and exchanging procedure. For the latter one, a paid model of sharing resource should be designed. For example, the computing and storage capability can be regarded as valuable resources, which can exchange with real educational information resources. It means that the more resources the education organization shared, the more computing and storage resources on the cloud it possess. This approach may simple the paid method and inspire the sharing behavior.

The third strategy is to design new methods to develop educational information resources in Cloud computing model. One approach is to provide resource authoring tools and personal resource storage center for every teacher. The educational resources will be created and shared in the course of nature during the teachers' normal education activities. Another approach is to use the resource discovery mechanism in cloud computing model to combing the resources on the Internet and local resources. These two methods will improve the efficiency of educational resource development.

\section{CONCLUSIONS}

The sharing and optimized configuration of educational information resources is very important of the realization of fair education. In this paper, we discussed with the apply of cloud computing to the sharing of educational resources. The way and the benefit of the cloud computing applying are very carefully investigated. Supplementary strategies of applying cloud computing to solve remained problems are also proposed.

We believe that how to enhance the advantages of cloud computing applying to educational information sharing would still an open issue in future research.

\section{ACKNOWLEDGMENT}

This work is supported by the Project named Research on Educational Information Resource Sharing and Optimal Configuration based on Cloud Computing granted by the
Humanities and Social Science project of Chinese Ministry of Education with Grant No. 11YJA880163.

\section{REFERENCES}

[1] Yang Yinfu, Idea and Policy Innovation on National Education Plan Educational Research[J],2010(8):3-12.

[2] Mell P,Grance T.The NIST Definition of Cloud Computing[R].

National Institute of Standards and Technology,Information Technology Laboratory, 2009.

[3] Qian Wenjing, Deng Zhonghua. Cloud Computing and Management of Information Resource Sharing [J]. Books and Information, 2009(4).

[4]Robert L. Grossman, Yunhong Gu, et al.Compute and storage clouds using wide area high performance networks [J] .Future Generation Computer Systems, 2009,(25):179-183.

[5] Jinzy Zhu. Smarter Cloud Computing [M].Beijing: Publishing House of Electronics Industry, 2012.

[6] Guo Leshen, Zhang Naijing, Shang Jingang. Cloud computing environment security framework.[J] Network Information Security, 2009,10(7):62-64 